UCT-TP $188 / 92$

$\mathrm{MZ}-\mathrm{TH} / 92-54$

November 1992

\title{
Spectral Functions for Heavy-Light Currents and Form Factor Relations in HQET
}

\author{
C.A. Dominguez" \\ Institute of Theoretical Physics and Astrophysics \\ University of Cape Town, Rondebosch 7700, Cape, RSA \\ and \\ J.G. Körner ${ }^{2}$ and K. Schilcher' \\ Institut für Physik, Johannes Gutenberg-Universität \\ Staudingerweg 7, D.6500 Mainz, Germany
}

\begin{abstract}
We derive relations among form factors describing the current-induced transitions: (vacuum) $\rightarrow B, B^{*}, B \pi, B^{*} \pi, B \rho$ and $B^{*} \rho$ using heavy quark symmetry. The results are compared to corresponding form factor relations following from identities between scalar and axial vector, and pseudoscalar and vector spectral functions in the heavy quark limit.
\end{abstract}

\footnotetext{
${ }^{1}$ Supported in part by the Foundation for Research Development, South Africa.

${ }^{2}$ Supported in part by Bundesministerium für Forschung und Technologie BMFT, FRG under contract 06MZ730 and by the Humboldt Foundation.
} 
With the introduction of the heavy quark effective theory [1], (HQET) we have witnessed dramatic developments in our understanding of the physics of hadrons containing a heavy quark Q. The systematic expansion in inverse powers of the heavy quark mass $m_{Q}$ accomplished in the HQET allows QCD calculations of hadronic processes to a level of rigor previously only conceivable in deep inelastic reactions. In the heavy quark limit (HQL) the effective Lagrangian exhibits a new spin-flavor symmetry [1]. This heavy quark symmetry (HQS) imposes restrictive constraints on weak decay amplitudes. Notable results are the scaling relation between decay constants [2] and the reduction of semileptonic form factors of heavy mesons and baryons to a small number of Isgur-Wise functions [1].

In this paper we will present an alternative approach to obtain relations between form factors in the heavy quark limit $(H Q L)$. Our approach is based on the observation that in this limit certain correlators of two currents comprised of a heavy and a light quark become identical (while they bear no relation to each other in the full QCD). For example, the vector-vector (VV) correlator equals the pseudoscalar-pseudoscalar (PP) one and the axial vector-axial vector (AA) correlator equals the scalar-scalar (SS) one. If we adopt the point of view that the physical spectral function is obtained from the QCD correlator by some form of analytic continuation, then identical QCD correlators imply identical physical spectral functions. We will try in this paper to exploit this form of duality as far as possible.

We consider two-point functions defined (in full QCD) through

$$
\Pi_{\mu \nu}(q)=i \int d x e^{i q x}\left\langle 0\left|T\left(J_{\mu}(x) J_{\nu}^{\dagger}(0)\right)\right| 0\right\rangle
$$

where the renormalized currents are

$$
J_{\mu}(x)=\bar{q}(x) \Gamma_{\mu} Q(x), \quad \Gamma_{\mu}=\gamma_{\mu} \text { or } \gamma_{\mu} \gamma_{5}
$$

with $q(x)(Q(x))$ being the light (heavy) quark field.

Given a hadron with four-velocity $v_{\mu}$ and assuming that the four-velocity of the heavy quark is almost equal to $v_{\mu}$ one can factorize out the large $m_{Q}$ effects by introducing a field $h_{v}(x)$ through

$$
h_{v}(x)=\frac{1+\not b}{2} e^{i m_{Q} v \cdot x} Q(x)
$$

which carries only the residual momentum. When the QCD Lagrangian is expressed in terms of the field $h_{v}(x)$ it exhibits the well-known static spin flavour symmetries.

The current (2) then goes over to

$$
J_{\mu}(x) \rightarrow e^{-i m_{Q} v \cdot x} \tilde{J}_{\mu}(x)
$$

where $\tilde{J}_{\mu}(x)$ is the current in the effective theory.

$$
\tilde{J}_{\mu}(x)=\bar{q}(x) \Gamma_{\mu} h_{v}(x)
$$


The matrix elements of $\tilde{J}_{\mu}$ satisfy the spin-flavor symmetries. The HQET predictions for decay parameters must, however, still be related to the physical (i.e. full QCD) ones through the procedure of running and matching.

The correlator in the effective theory reads

$$
\begin{aligned}
\tilde{\Pi}_{\mu \nu}(q) & =i \int d x e^{i q x}\left\langle 0\left|T\left(\tilde{J}_{\mu}(x) \tilde{J}_{\nu}^{\dagger}(0)\right)\right| 0\right\rangle \\
& =\left(-g_{\mu \nu} q^{2}+q_{\mu} q_{\nu}\right) \tilde{\Pi}^{(1)}\left(q^{2}\right)+q_{\mu} q_{\nu} \tilde{\Pi}^{(0)}\left(q^{2}\right)
\end{aligned}
$$

In $H Q E T$ the following symmetry relations hold

$$
\begin{aligned}
\tilde{\Pi}_{V V}^{(1)} & =\tilde{\Pi}_{A A}^{(0)} \\
\tilde{\Pi}_{A A}^{(1)} & =\tilde{\Pi}_{V V}^{(0)}
\end{aligned}
$$

where we emphasize that the currents in the correlators (6) are HQET currents. The subscripts $V V$ and $A A$ refer to the currents involved in the correlator (6).

The absorptive part of the two-point function is given by

$$
\begin{aligned}
\operatorname{Im} \tilde{\Pi}_{\mu \nu}(q)= & (2 \pi)^{3} \int d x e^{i q x} \sum_{n} \delta\left(q-p_{n}\right) \times\left\langle 0\left|\tilde{J}_{\mu}(0)\right| n\right\rangle\left\langle n\left|\tilde{J}_{\nu}^{\dagger}(0)\right| 0\right\rangle \\
& \equiv\left(-g_{\mu \nu} q^{2}+q_{\mu} q_{\nu}\right) \rho^{(1)}\left(q^{2}\right)+q_{\mu} q_{\nu} \rho^{(0)}\left(q^{2}\right)
\end{aligned}
$$

Depending on the parity of the current $\tilde{J}_{\mu}$, different intermediate states $n$ contribute to the spectral functions $\rho^{(i)}(i=0,1)$. The most important intermediate states which we will consider in the following are listed in Table 1.

Table 1: Lowest lying intermediate states contributing to the spectral functions $\rho_{V V}^{(i)}$ and $\rho_{A A}^{(i)}(i=0,1)$.

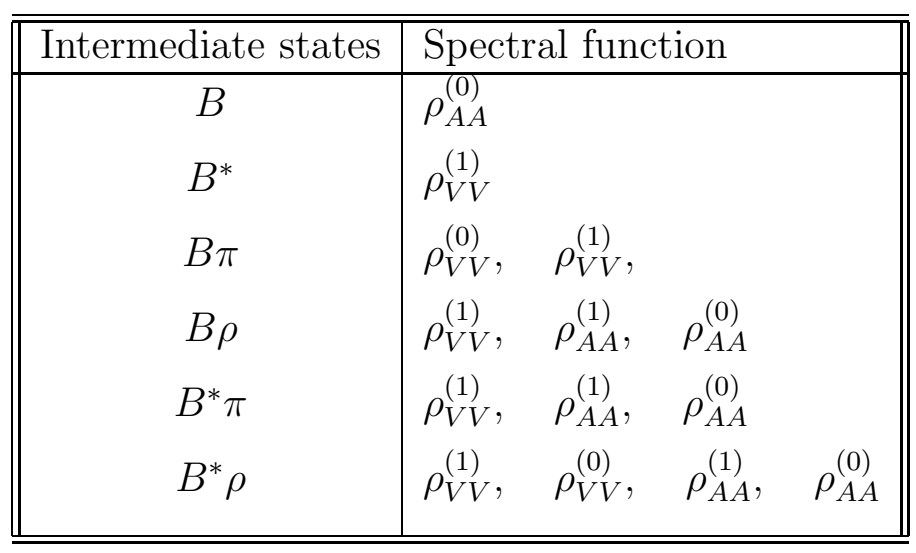

In the HQET [3]

$$
\rho_{V V}^{(1)}\left(q^{2}\right)=\rho_{A A}^{(0)}\left(q^{2}\right)
$$




$$
\rho_{A A}^{(1)}\left(q^{2}\right)=\rho_{V V}^{(0)}\left(q^{2}\right)
$$

for $q^{2}$ close to the heavy-light threshold satisfying $\left(q^{2}-m_{Q}^{2}\right)<<m_{Q}^{2}$. From the identity Eq.(9) one immediately concludes the equality of the leptonic decay constants $f_{B}$ and $f_{B^{*}}$ as a manifestation of the spin symmetry, where the decay constants are defined as

$$
\begin{aligned}
\left\langle 0\left|A_{\mu}\right| B\right\rangle & =i f_{B} p_{\mu} \\
\left\langle 0\left|V_{\mu}\right| B^{*}\right\rangle & =f_{B^{*}} M_{B^{*}} \epsilon_{\mu}
\end{aligned}
$$

For the two particle intermediate states we expect relations between various form factors. To determine these we first define the relevant matrix elements,

$$
\begin{aligned}
\left\langle B \pi\left|V_{\mu}\right| 0\right\rangle= & \sqrt{M_{1}}\left(f_{1}^{V} v_{1 \mu}-f_{2}^{V} v_{2 \mu}\right) \\
\left\langle B^{*} \pi\left|V_{\mu}\right| 0\right\rangle= & \sqrt{M_{1}} f^{* V} i \epsilon\left(\mu \epsilon_{1}^{*} v_{1} v_{2}\right) \\
\left\langle B^{*} \pi\left|A_{\mu}\right| 0\right\rangle= & \sqrt{M_{1}} \epsilon_{1}^{* \alpha}\left[f_{1}^{* A} g_{\alpha \mu}+f_{2}^{* A} v_{2 \alpha} v_{1 \mu}+f_{3}^{* A} v_{2 \alpha} v_{2 \mu}\right] \\
\left\langle B \rho\left|V_{\mu}\right| 0\right\rangle= & \sqrt{M_{1}} g^{V} i \epsilon\left(\mu \epsilon_{2}^{*} v_{1} v_{2}\right) \\
\left\langle B \rho\left|A_{\mu}\right| 0\right\rangle= & \sqrt{M_{1}} \epsilon_{2}^{* \alpha}\left[g_{1}^{A} g_{\alpha \mu}+g_{2}^{A} v_{1 \alpha} v_{1 \mu}+g_{3}^{A} v_{1_{\alpha}} v_{2_{\mu}}\right] \\
\left\langle B^{*} \rho\left|V_{\mu}\right| 0\right\rangle= & \sqrt{M_{1}}\left[\epsilon_{1 \alpha}^{*} \epsilon_{2 \beta}^{*} v_{1 \beta} v_{2 \alpha}\left(v_{1 \mu} g_{1}^{* V}+v_{2 \mu} g_{2}^{* V}\right)\right. \\
& \left.+g_{\alpha \beta}\left(v_{1 \mu} g_{3}^{* V}+v_{2 \mu} g_{4}^{* V}\right)+g_{\alpha \mu} v_{1 \beta} g_{5}^{* V}+g_{\beta \mu} v_{2 \alpha} g_{6}^{* V}\right] \\
\left\langle B^{*} \rho\left|A_{\mu}\right| 0\right\rangle= & \sqrt{M_{1}}\left[i \epsilon\left(\mu \epsilon_{1}^{*} \epsilon_{2}^{*} v_{1}\right) g_{1}^{* A}+i \epsilon\left(\mu \epsilon_{1}^{*} \epsilon_{2}^{*} v_{2}\right) g_{2}^{* A}\right. \\
& \left.+i \epsilon\left(\mu \epsilon_{1}^{*} v_{1} v_{2}\right) \epsilon_{2}^{*} v_{1} g_{3}^{* A}+i \epsilon\left(\mu \epsilon_{2}^{*} v_{1} v_{2}\right) \epsilon_{1}^{*} v_{2} g_{4}^{* A}\right]
\end{aligned}
$$

where the indices 1 and 2 refer to the heavy and light meson, respectively. The normalization of the heavy meson state is chosen to be E/M to facilitate comparison with the $H Q L$. We use velocities $v_{i}=p_{i} / M_{i}(i=1,2)$ rather than momenta in the definition of the form factors so that they do not depend on the heavy mass scale.

In the HQET the form factor complexity is reduced. Denoting the HQET heavy to light reduced form factors by capital letters we obtain the following results using the well-known trace formalism [1] as applied to heavy meson to light meson transitions (see also [4])

$$
\begin{aligned}
\left\langle B \pi\left|V_{\mu}\right| 0\right\rangle & =\sqrt{M_{1}}\left(F_{1} v_{1 \mu}-F_{2} v_{2 \mu}\right) \\
\left\langle B^{*} \pi\left|V_{\mu}\right| 0\right\rangle & =\sqrt{M_{1}}\left(F_{2} i \epsilon\left(\mu \epsilon_{1}^{*} v_{1} v_{2}\right)\right) \\
\left\langle B^{*} \pi\left|A_{\mu}\right| 0\right\rangle & =\sqrt{M_{1}}\left(\left(F_{1}-\omega F_{2}\right) \epsilon_{1 \mu}^{*}+F_{2}\left(v_{2} \epsilon_{1}^{*}\right) v_{1 \mu}\right) \\
\left\langle B \rho\left|V_{\mu}\right| 0\right\rangle & =\sqrt{M_{1}}\left(-G_{1}-G_{3}\right) i \epsilon\left(\mu \epsilon_{2}^{*} v_{1} v_{2}\right) \\
\left\langle B \rho\left|A_{\mu}\right| 0\right\rangle & =\sqrt{M_{1}}\left\{\left(\omega G_{1}-G_{2}-\omega G_{3}+G_{4}\right) \epsilon_{2 \mu}^{*}-2 G_{4} v_{1} \epsilon_{2}^{*} v_{1 \mu}+\left(-G_{1}+G_{3}\right)\left(v \cdot \epsilon_{2}^{*}\right) v_{2 \mu}\right\}
\end{aligned}
$$




$$
\begin{aligned}
\left\langle B^{*} \rho\left|V_{\mu}\right| 0\right\rangle= & \sqrt{M_{1}}\left\{-2 G_{3} v_{1 \mu}\left(v_{1} \cdot \epsilon_{2}^{*}\right)\left(v_{2} \cdot \epsilon_{1}^{*}\right)+\left(G_{2}+2 \omega G_{3}-G_{4}\right)\left(\epsilon_{2}^{*} \cdot \epsilon_{1}^{*}\right) v_{1 \mu}\right. \\
& \left.+\left(G_{1}+G_{3}\right)\left[\left(v_{2} \cdot \epsilon_{1}^{*}\right) \epsilon_{2 \mu}^{*}-\left(\epsilon_{1}^{*} \cdot \epsilon_{2}^{*}\right) v_{2 \mu}\right]-\left(G_{2}+G_{4}\right)\left(v_{1} \cdot \epsilon_{1 \mu}^{*}\right)\right\} \\
\left\langle B^{*} \rho\left|A_{\mu}\right| 0\right\rangle= & \sqrt{M_{1}}\left\{\left(-G_{2}-2 w G_{3}+G_{4}\right) i \epsilon\left(\mu \epsilon_{1}^{*} \epsilon_{2}^{*} v_{1}\right)\right. \\
& \left.+\left(G_{1}+G_{3}\right) i \epsilon\left(\mu \epsilon_{1}^{*} \epsilon_{2}^{*} v_{2}\right)-2 G_{3}\left(v_{1} \cdot \epsilon_{2}^{*}\right) i \epsilon\left(\mu \epsilon_{1}^{*} v_{1} v_{2}\right)\right\}
\end{aligned}
$$

where $\omega=\left(q^{2}-M_{1}^{2}-M_{2}^{2}\right) / 2 M_{1} M_{2}$. Some of these results have been derived before [41. As a next task we would like to demonstrate how Eqs.(12) and (13) follow from the equalities between spectral functions Eqs.(9) and (10), or are consistent with them.

In order to be able to express our results in a compact form we will use helicity form factors rather than the covariant ones of Eq.(12). In the CM system with z-axis in the direction of the outgoing heavy particle the polarization vectors $\epsilon_{\mu}\left(\lambda_{W}\right)$ associated with the currents are

$$
\begin{aligned}
\epsilon_{\mu}(0) & =(0,0,0,1), \quad \epsilon_{\mu}( \pm)=\frac{1}{\sqrt{2}}(0, \mp 1,-i, 0) \\
\epsilon_{\mu}(t) & =(1,0,0,0)
\end{aligned}
$$

where $\epsilon_{\mu}(0, \pm)$ and $\epsilon_{\mu}(t)$ refer to the spin 1 and spin 0 components of the currents, respectively.

We consider first the $B \pi$ intermediate states. The helicity form factors are defined through

$$
H_{\lambda_{W}}^{V}=\left\langle B \pi\left|V_{\mu}\right| 0\right\rangle \cdot \epsilon^{\mu}\left(\lambda_{W}\right)
$$

Using Eqs.(12) and (14) one obtains

$$
\begin{aligned}
& \sqrt{q^{2}} H_{0}^{V}=\sqrt{M_{1}} \sqrt{\omega^{2}-1}\left(-M_{2} f_{1}^{V}+M_{1} f_{2}^{V}\right) \\
& \sqrt{q^{2}} H_{t}^{V}=\sqrt{M_{1}}\left(\left(M_{1}+M_{2} \omega\right) f_{1}^{V}+\left(M_{2}+M_{1} \omega\right) f_{2}^{V}\right) .
\end{aligned}
$$

In the $H Q L\left(M_{1} \rightarrow \infty\right.$ with $\omega$ fixed, i.e $\left.\sqrt{q^{2}} \simeq M_{1}\right)$ Eq.(16) reduces to

$$
\begin{aligned}
& H_{0}^{V}=-\sqrt{M_{1}} \sqrt{\omega^{2}-1} f_{2}^{V} \\
& H_{t}^{V}=\sqrt{M_{1}}\left(f_{1}^{V}+\omega f_{2}^{V}\right)
\end{aligned}
$$

since $f_{1}^{V}$ and $f_{2}^{V}$ are heavy mass scale independent.

This result is of the same form as the HQET relation Eq.(13). 
Next we turn to the $B^{*} \pi$ intermediate state. The helicity amplitudes for the $B^{*} \pi$ intermediate state are defined through

$$
H_{\lambda_{W}=\lambda_{1}}^{A}=\left\langle B^{*}\left(\lambda_{1}\right), \pi\left|A_{\mu}\right| 0\right\rangle \epsilon^{\mu}\left(\lambda_{W}\right)
$$

In the $H Q L$ one obtains

$$
\begin{aligned}
& H_{0}^{A}=\sqrt{\omega^{2}-1} \sqrt{M_{1}}\left[-f_{1}^{* A}+\left(\omega^{2}-1\right) f_{3}^{* A}\right] \\
& H_{t}^{A}=\sqrt{\omega^{2}-1} \sqrt{M_{1}}\left[f_{2}^{* A}+\omega f_{3}^{* A}\right] \\
& H_{ \pm}^{A}=-\sqrt{M_{1}} f_{1}^{* A} \\
& H_{ \pm}^{V}=\mp \sqrt{M_{1}} \sqrt{\omega^{2}-1} f^{* V} .
\end{aligned}
$$

The condition $\rho_{V V}^{(0)}=\rho_{A A}^{(1)}$ now reads

$$
3\left|H_{t}^{V}(B \pi)\right|^{2}=\left|H_{0}^{A}\left(B^{*} \pi\right)\right|^{2}+\left|H_{+}^{A}\left(B^{*} \pi\right)\right|^{2}+\left|H_{-}^{A}\left(B^{*} \pi\right)\right|^{2}
$$

or

$$
3\left|f_{1}^{V}+\omega f_{2}^{V}\right|^{2}=\left|f_{1}^{* A}-\left(\omega^{2}-1\right) f_{3}^{* A}\right|^{2}+2\left|f_{1}^{* A}\right|^{2}
$$

If we assume pole behaviour of the helicity form factors a solution of the equation (20) or $(21)$ is

$$
f_{3}^{* A}=0, \quad f_{1}^{* A}=f_{1}^{V}+\omega f_{2}^{V}
$$

Similarly, one has from $\rho_{A A}^{(1)}=\rho_{V V}^{(0)}$

$$
3\left|H_{t}^{A}\left(B^{*} \pi\right)\right|^{2}=\left|H_{0}^{V}(B \pi)\right|^{2}+\left|H_{+}^{V}\left(B^{*} \pi\right)\right|^{2}+\left|H_{-}^{V}\left(B^{*} \pi\right)\right|^{2}
$$

or

$$
3\left(\omega^{2}-1\right)\left|f_{2}^{* A}-\omega f_{3}^{* A}\right|^{2}=\left(\omega^{2}-1\right)\left|f_{2}^{V}\right|^{2}+2\left(\omega^{2}-1\right)\left|f^{* V}\right|^{2}
$$

Assuming again pole behaviour of the helicity form factors we obtain using Eq. (24) and the first relation in Eq.(22):

$$
f_{2}^{V}=-f_{2}^{* A}=-f^{* V}
$$

Eqs. (22) and (25) can be seen to be the HQET result Eq. (13) with $f_{1}^{V}=F_{1}$ and $f_{2}^{V}=-F_{2}$. They do not represent a unique solution and strictly speaking, we can only claim that the HQL spectral function identities Eqs.(9) and (10) are consistent with the explicit form factor results following from the HQL. Imposing pole dominance of the form factors, however, the HQL relations follow. 
Finally, we consider the $B \rho$ and $B^{*} \rho$ intermediate states. The helicity amplitudes for the $B \rho$ case are defined in analogy to Eq. (18) . The helicity amplitudes for $B^{*} \rho^{*}$ are defined by

$$
H_{\lambda_{W} ; \lambda_{B^{*}, \lambda_{\rho}}}=\left\langle 0\left|J_{\mu}\right| B^{*}\left(\lambda_{B^{*}}\right) \rho\left(\lambda_{\rho}\right)\right\rangle \epsilon^{\mu}\left(\lambda_{W}\right)
$$

with $\lambda_{W}=\lambda_{B^{*}}-\lambda_{\rho}$. The helicity amplitude expressions can be worked out but are too lenghty to be reproduced here.

The equality $\rho_{V V}^{(0)}=\rho_{A A}^{(1)}$ for the $B \rho$ and $B^{*} \rho$ system then implies

$$
\begin{aligned}
3\left|H_{t ; 00}^{V}\left(B^{*} \rho\right)\right|^{2}+6\left|H_{t ;++}^{V}\left(B^{*} \rho\right)\right|^{2}= & 2\left|H_{+; 0-}^{A}(B \rho)\right|^{2}+2\left|H_{+;+0}^{A}\left(B^{*} \rho\right)\right|^{2}+2\left|H_{+; 0-}^{A}\left(B^{*} \rho\right)\right|^{2} \\
& +\left|H_{0 ; 00}^{A}(B \rho)\right|^{2}+2\left|H_{0 ;++}^{A}\left(B^{*} \rho\right)\right|^{2}
\end{aligned}
$$

Similarly, it follows from $\rho_{A A}^{(0)}=\rho_{V V}^{(1)}$ that

$$
\begin{aligned}
3\left|H_{t ; 00}^{A}(B \rho)\right|^{2}+6\left|H_{t ;++}^{A}\left(B^{*} \rho\right)\right|^{2}= & 2\left|H_{+; 0-}^{V}(B \rho)\right|^{2}+2\left|H_{+;+0}^{V}\left(B^{*} \rho\right)\right|^{2}+2\left|H_{+; 0-}^{V}\left(B^{*} \rho\right)\right|^{2} \\
& +\left|H_{0 ; 00}^{V}\left(B^{*} \rho\right)\right|^{2}+2\left|H_{0 ;++}^{V}\left(B^{*} \rho\right)\right|^{2}
\end{aligned}
$$

The HQL results in Eq.(13) can be seen to satisfy Eqs. (27) and (28). It seems to be impossible, however, to derive the HQL results from these two equations as they involve altogether ten form factors. All we can say is that the spectral function identities are consistent with the HQL form factors.

In conclusion we have derived relations among form factors describing the current-induced transitions: (vacuum) $\rightarrow B, B^{*}, B \pi, B^{*} \pi, B \rho$ and $B^{*} \rho$ in the HQET. We show that many of these form factor relations may be obtained in a simple manner by exploiting the identities between the scalar and axial vector, and pseudoscalar and vector spectral functions which hold in the heavy quark limit by considering in turn the various one- and two-particle intermediate states.

\section{Acknowledgement}

Part of this work was done while two of us (JGK and KS) were visitors at the University of Cape Town, Republic of South Africa. JGK and KS would like to thank the Institute of Theoretical Physics at UCT for their hospitality.

\section{References}

[1] N. Isgur and M.B. Wise, Phys Lett B237 (1990) 527; J.D. Bjorken, invited talk at Les Recontres de Physique de la Vall'ee d'Aosta, La Thuile, Italy, SLAC-PUB-5278 (1990); A. Falk, H. Georgi, B. Grinstein and M.B. Wise, Nucl Phys B343 (1990) 1; B. Grinstein, Nucl Phys B339 (1990) 253; H. Georgi, Phys Lett B240 (1990) 447; 
E. Eichten and B. Hill, Phys Lett B240 (1990) 511; F. Hussain, J.G. Körner, K. Schilcher, G. Thompson and Y.L. Wu, Phys Lett B249 (1990) 295.

[2] M.B. Voloshin and M.A. Shifman, Sov.J.Nucl.Phys. 47 (1988) 511; H.D. Politzer and M.B. Wise, Phys Lett B206 (1988) 68.

[3] C.A.Dominguez,N.Paver,Phys.Lett.B 269 (1991) 169; ibid. B 276 (1992) 179.

[4] G. Kramer, T. Mannel and G.A. Schuler, Z.Phys. C51 (1991) 649. 